\title{
Dynamics of the Molecules in Solid Phases of Few Chosen Schiff Bases Compounds
}

\author{
A. BAqK ${ }^{a, *}$ AND M. MAssalska-Arodź ${ }^{b}$ \\ ${ }^{a}$ Chair of Physics, Technical University of Rzeszów \\ Pola 2, 39-959 Rzeszów, Poland \\ ${ }^{b}$ Henryk Niewodniczański Institute of Nuclear Physics \\ Radzikowskiego 152, 31-342 Kraków, Poland
}

(Received April 19, 2004)

\begin{abstract}
The dielectric relaxation processes observed in solid phases of MBBA, OH-MBBA, and EBBA belonging to the homologous series of the Schiff bases are discussed. The data are compared with the results completed by the present authors for EBPA compound. The uniform interpretation of the temperature changes of relaxation for all mentioned compounds is proposed.
\end{abstract}

PACS numbers: $64.70 . \mathrm{Md}, 77.22 . \mathrm{Gm}$

\section{Introduction}

The Schiff bases are liquid crystalline compounds with the chemical structure of the molecule shown in Fig. 1. Due to the dipolar moment of the $-\mathrm{CH}=\mathrm{N}-$ bridge group all compounds are polar regardless of the $\mathrm{R}$ and $\mathrm{R}^{\prime}$ substituents forms. Polymorphism of each compound depends on the cooling rate used but some common features of phase diagram can be traced [1,2]. Generally, on slow cooling of the nematic phase $\mathrm{N}$ one or two crystalline phases were observed while on fast cooling the original nematic phase is frozen into glass (MBBA and OH-MBBA), or the transition to the smectic phase takes place (EBBA). On subsequent heating a rich solid state polymorphism was found for each compound. Number of the solid phases observed on heating is larger for higher cooling rates (MBBA $-5 / 2$, OH-MBBA $-4 / 1$, EBBA $-3 / 2$. The first digit means a number of solid phases observed during heating process after fast cooling, whereas the next one means the number of phases during a slow cooling of a sample). Moreover, for all investigated

* corresponding author; e-mail: sowa@prz.rzeszow.pl 


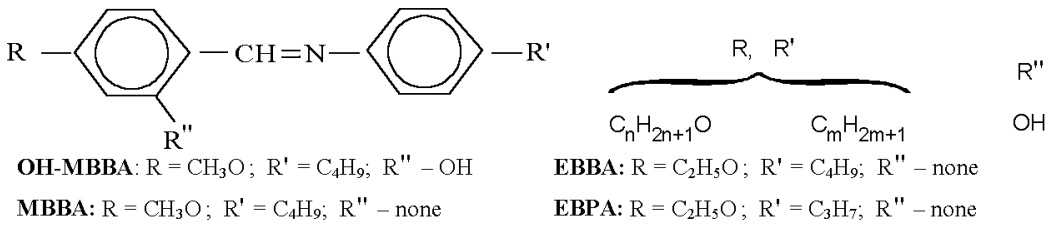

Fig. 1. Scheme of the molecule belonging to homologous series Shiff's bases and the possible substituents.

compounds one (in OH-MBBA and $\mathrm{EBBA}$ ) or two (in MBBA) phases with layer order of the molecules (smectic phases) were found when the cooling process was rapid.

The aim of the paper is to present the main results of the dielectric relaxation observed in solid phases of several Schiff bases compounds and to compare them with those obtained by the authors for EBPA in order to formulate the uniform interpretation of the dynamics of molecules.

\section{Dielectric relaxation in solid phases for MBBA, OH-MBBA, and EBBA}

\section{1. $M B B A$}

The complex electric permittivity $\varepsilon^{*}(f, T)=\varepsilon^{\prime}(f, T)-\mathrm{i} \varepsilon^{\prime \prime}(f, T)$ in metastable and stable phases of MBBA was first measured by Mościcki [3] (see Fig. 2) and by Mościcki and Urban [4]. The authors tried to find a reason of the specific heat anomaly observed earlier in calorimetric measurement [5] in the metastable phase. It was found that the dielectric absorption appears only in the metastable solid phase and it is caused by the relaxation of the methoxy groups reorienting round the $\mathrm{C}-\mathrm{O}$ bounds. The observed $\varepsilon^{\prime \prime}(f)$ spectrum was described by the Cole-Davidson equation and the authors suggested that it is probably a consequence of two overlapping processes, i.e. the reorientations of the methoxy groups and of the butyl chains. The value of the Kirkwood dipole-dipole correlation factor $g<1$ pointed to the antiparallel arrangement of the MBBA molecules in the metastable phase. Therefore, methoxy group reorientations are possible if only a sufficient room is created by conformational changes of the butyl chains of the neighboring molecules.

For the quantitative description of the relaxation the Glarum model [4] has been used. One can see that at temperatures in which the double peak maximum was found in the calorimetric measurements the changes of such parameters as $\tau_{\mathrm{r}}, \beta$, and $\tau_{\mathrm{d}} / \tau_{\mathrm{r}}$ were detected. $\tau_{\mathrm{r}}$ means the relaxation time characterizing the reorientations of the methoxy groups. The $\beta$ parameter describes a distribution of the relaxation time and corresponds to the broadening of the absorption $\varepsilon^{\prime \prime}(f)$ curve, while $\tau_{\mathrm{d}}$ is the relaxation time of the defect diffusion connected with the conformational changes of the butyl chains. It was found that terminal groups in 


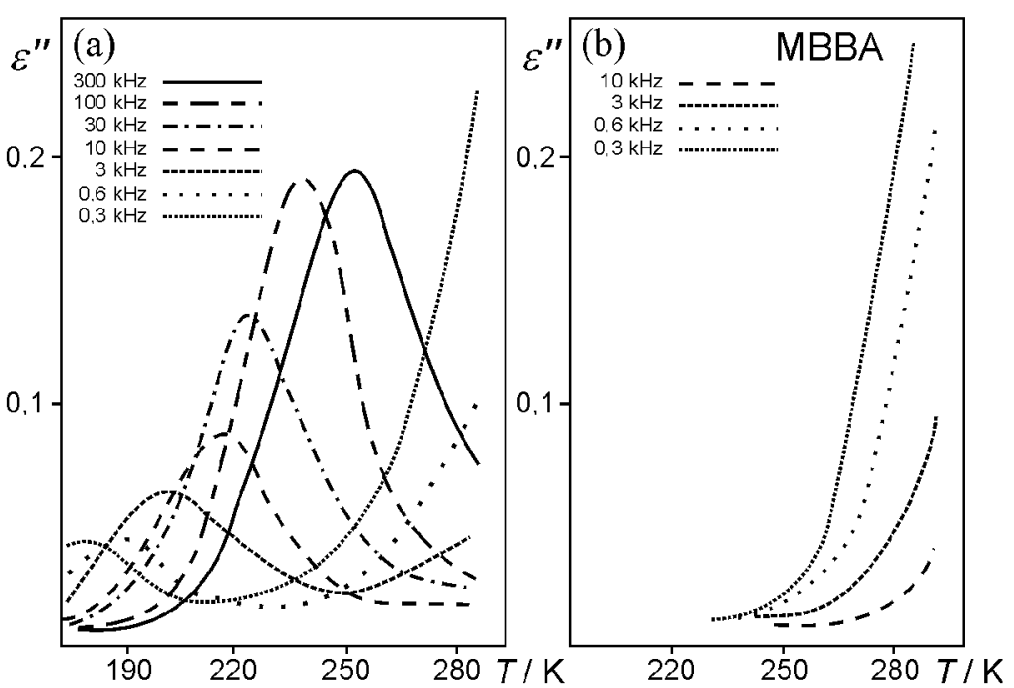

Fig. 2. Temperature dependence of the dielectric losses $\varepsilon^{\prime \prime}$ for two modifications of the solid phase of MBBA (a) metastable, (b) stable, for various frequencies (based on data from [3]).

both molecular chains are mobile also below the temperature where the specific heat anomaly was observed. Therefore, the wider component of the specific heat double peak anomaly was attributed to the fact that butyl chain conformational changes gain freedom and second component to the appearance of the methoxy groups rotation.

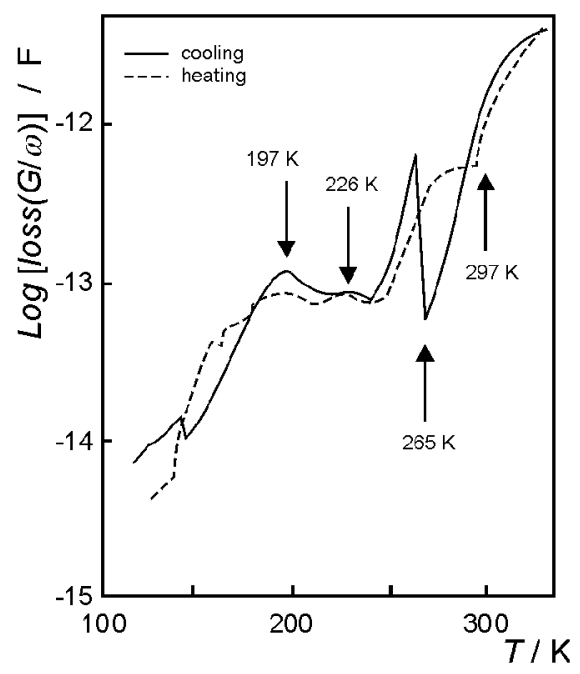

Fig. 3. The dielectric response observed at $1.78 \mathrm{kHz}$ during slow cooling of the isotropic phase and on subsequent heating (based on data from [6]). 
Dielectric relaxation processes in the solid phases of MBBA were investigated also by Pathmanathan et al. [6]. They analysed the temperature dependence of $\varepsilon^{*}(f)$ for the samples in three conditions, i.e. during heating of the solid phases formed in slow cooling $(1 \mathrm{~K} / \mathrm{min})$ of the nematic and of the isotropic phase, and also during heating of the phase formed in fast cooling $(3.5 \mathrm{~K} / \mathrm{min})$ of a nematic. The dielectric absorption and dispersion was observed in each case which means that the stable form of MBBA that was studied by Mościcki and Urban was not detected in their experiment.

As one can see in Fig. 3 on heating, Pathmanathan et al. observed two regions of absorption. One of them with significant intensity reaches a maximum value at the temperature about $270 \mathrm{~K}$. This effect was attributed by Mościcki and Urban to the relaxation connected with reorientations of the methoxy groups round the $\mathrm{C}-\mathrm{O}$ bounds. However, the data received by Pathmanathan were approximated by the Cole-Cole function, whereas Mościcki and Urban data were approximated by the Cole-Davidson function. Second absorption, which appeared in the range of temperature from 198 to $213 \mathrm{~K}$ and disappeared at $228 \mathrm{~K}$ was attributed to the motions of the benzene cores leading to better packing of molecular structure of the sample and to larger sterical difficulties for reorientations.

\section{2. $O H-M B B A$}

Arora et al. [7] performed the dielectric measurements for solid phase in OH-MBBA. The samples were cooled in two ways: a fast one - with the cooling
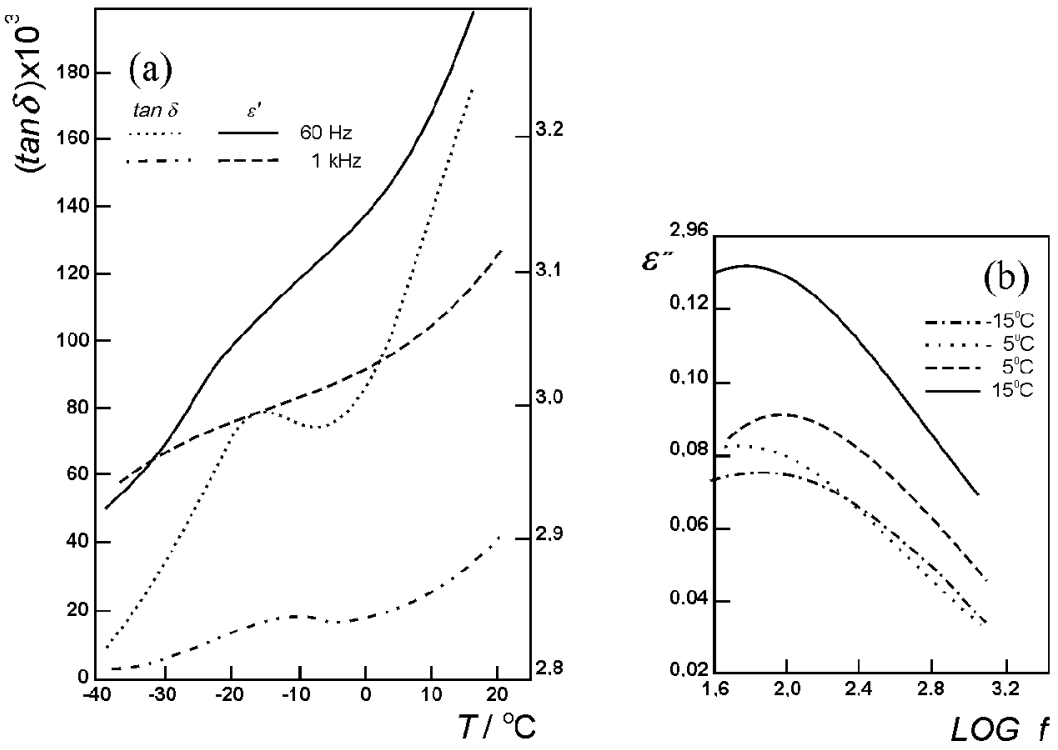

Fig. 4. (a) Temperature dependence of dielectric loss factor $(\tan \delta)$ and dielectric permittivity $\left(\varepsilon^{\prime}\right)$ for the solid phase of OH-MBBA; (b) frequency dependence of the dielectric loss $\varepsilon^{\prime \prime}$ for several temperatures of the solid phase OH-MBBA (based on data from [7]). 
rate of $8 \mathrm{~K} / \mathrm{min}$, and a slow one - with the cooling rate of $0.5 \mathrm{~K} / \mathrm{min}$. The relaxation phenomena were observed in both cases. The dielectric dispersion and absorption curves in the metastable and the stable phases have similar shapes and the dielectric parameters $\Delta \varepsilon$ and $\tau$ have the comparable values. The changes of $\varepsilon^{\prime}(T)$ and $\tan \delta=\varepsilon^{\prime \prime}(T) / \varepsilon^{\prime}(T)$ values measured at $60 \mathrm{~Hz}$ and $1 \mathrm{kHz}$ are shown in Fig. 4a. In Fig. $4 \mathrm{~b}$ there are presented the values $\varepsilon^{\prime \prime}(f)$ for temperatures of $-15^{\circ} \mathrm{C}$, $-5^{\circ} \mathrm{C}, 5^{\circ} \mathrm{C}$ and $15^{\circ} \mathrm{C}$. In both cases the absorption occurring at low temperatures was attributed to the rotations of methoxy groups round the $\mathrm{C}-\mathrm{O}$ bonds. The increase in temperature up to $5^{\circ} \mathrm{C}$ shifted the maximum of $\varepsilon^{\prime \prime}(f)$ up to $131 \mathrm{~Hz}$ (see Fig. 4b). On further heating surprisingly the shift of the maximum back to lower frequencies was observed (at $15^{\circ} \mathrm{C}, f_{\max }=50 \mathrm{~Hz}$ ). Probably, at the temperature $0^{\circ} \mathrm{C}$, the phase transition occurs in which the whole molecules gain freedom to rotate round the principal axis.

\section{3. $E B B A$}

The first dielectric measurements of EBBA were performed by Agarwal and Arora [8]. Depending on a cooling rate of the sample they received the metastable (cooling rate $-5 \mathrm{~K} / \mathrm{min}$ ) or stable (cooling rate $-0.5 \mathrm{~K} / \mathrm{min}$ ) modification of a crystalline phase. Measurements of the electric permittivity $\varepsilon^{\prime}(T)$ and the dielectric loss coefficient $\tan \delta(T)$ were performed for five chosen frequencies in the range of $0.1 \mathrm{kHz}$ to $100 \mathrm{kHz}$ during heating the samples in the temperature range from $-170^{\circ} \mathrm{C}$ to $-10^{\circ} \mathrm{C}$. As for $\mathrm{MBBA}$, the dielectric relaxation was observed only in the metastable phase (see Fig. 5) in the form of two clear regions of dielectric

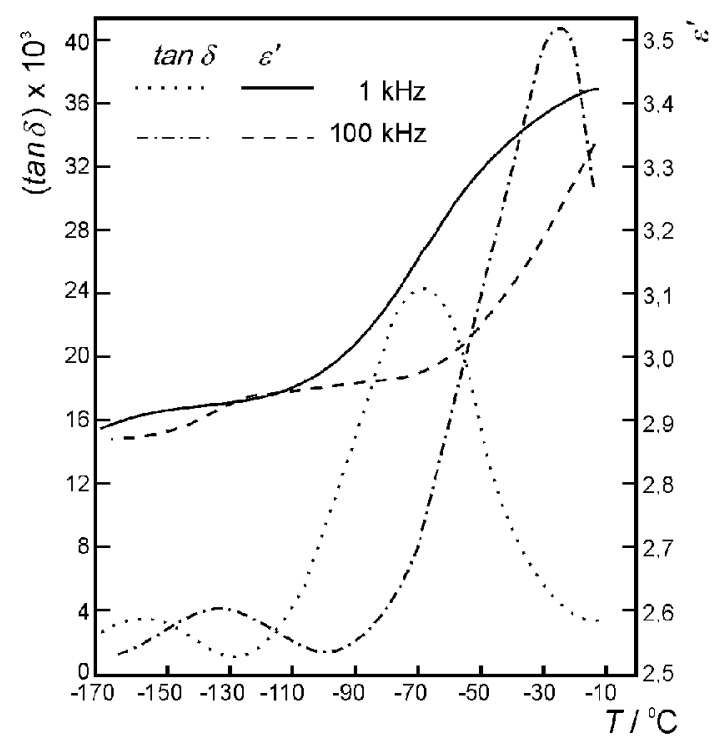

Fig. 5. Temperature dependence of dielectric loss factor $(\tan \delta)$ and dielectric permittivity $\left(\varepsilon^{\prime}\right)$ for the metastable solid phase of EBBA (based on data from [8]). 
absorption. This regions were explained by the processes of an $\alpha$ and $\beta$ relaxation appearing due to the transformation of the nematic phase into glass.

\subsection{Summary}

Interpretation of the $\varepsilon^{*}(f, T)$ results received for the solid phases of MBBA, $\mathrm{OH}-\mathrm{MBBA}$, and EBBA given by several authors is ambiguous. Comparing their papers one can find just few essential differences. The first one concerns dynamics of molecules in the stable phase of MBBA. Mościcki and Urban [4] found that molecules are mobile only in metastable phase, while Pathmanatan et al. did not observe a stable phase without dynamics of molecules which must be due to a different thermal history of the samples used in their studies. Next, evident difference concerns the model of relaxation used to describe the results as Mościcki and Urban used the Cole-Davidson function whereas Pathmanathan et al. the Cole-Cole function. There is also a discrepancy in interpretation of the dynamics responsible for the relaxation observed: Mościcki and Urban registered just one region of relaxation ascribed to the methoxy groups rotation around $\mathrm{CO}$ bonds while Pathmanathan et al. additionally observed the smaller absorption, ascribed to reorientations of the rigid benzene core. Next, the observed spectrum for OH-MBBA is explained in the opposite way to the proposed by Pathmanathan et al. [6] for MBBA, i.e. the small maximum of $\varepsilon^{\prime \prime}(f)$ was ascribed to the methoxyl group rotation while the more intensive one, to the reorientation of molecules round the principal axis.

There are also contradictions in interpretation of the data for EBBA. Agarwal et al. [8] assumed that during cooling with the rate of $8 \mathrm{~K} / \mathrm{min}$ only a glass

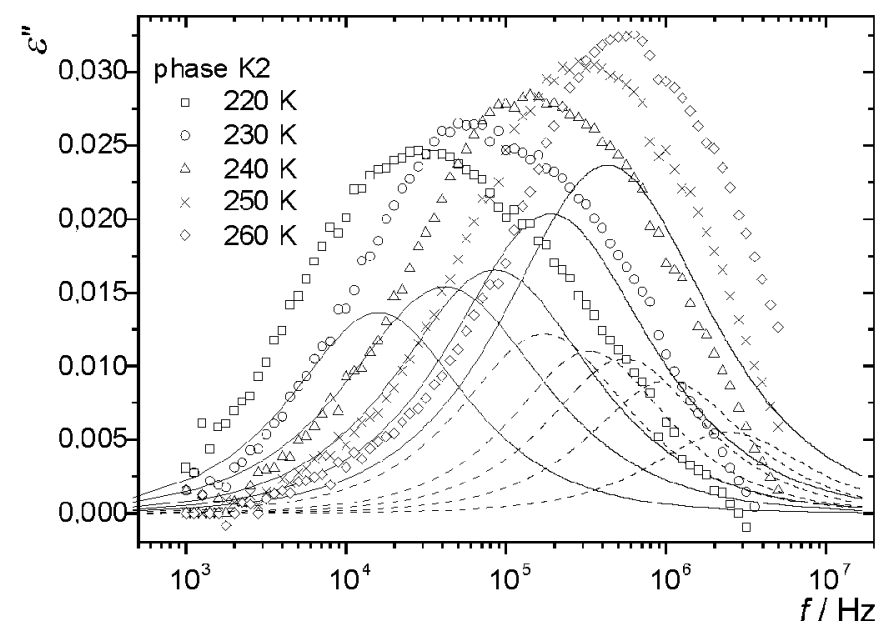

Fig. 6. Frequency dependence of imaginary part of permittivity during heating of stable phase of EBPA. Solid and dashed lines correspond to the first and second components of the fitting procedure. 
with the $\alpha$ and $\beta$ relaxations occurs, while Fouret et al. [2] did not confirm this hypothesis even using very high cooling rates, about $-200 \mathrm{~K} / \mathrm{min}$.

Thus despite the fact that for MBBA, OH-MBBA, and EBBA compounds the dielectric loss curves versus temperature (Figs. 3, 4 and 6) are similar, their interpretation proposed by the authors is different.

\section{Dynamics in the stable solid phase of EBPA}

Quantitative description of a dielectric relaxation in the solid phases of EBPA was shown by Bak et al. [9]. The relaxation process was found in the metastable and the stable phases. An essential feature of the observed spectra was their large width. Analysis of the received data of $\varepsilon^{*}(f, T)$ shows that the measuring points are well described by the sum of two Cole-Cole function. The $\varepsilon^{\prime \prime}(f, T)$ data for the stable phase are shown in Fig. 6 together with the results of the fitting procedure.

The main process occurring at the low frequencies was ascribed to reorientations of ethoxy groups round $\mathrm{C}-\mathrm{O}$ bonds, while the smaller one to rotations of the whole molecules round the long axis. This assignment is based on the analysis of the temperature dependence of the $\varepsilon_{\max 1}^{\prime \prime}(T)$ and $\varepsilon_{\max 2}^{\prime \prime}(T)$ components which is different. The amplitude of the first process increases with temperature, whereas the second process is gradually disappearing. The fact that this absorption was observed at the narrow interval of temperature means that this motion meets difficulties which increase on heating.

Interpretation of the results of $\varepsilon^{*}(f, T)$ is in agreement with that proposed by Pathmanathan et al. [6] for MBBA where the relaxation processes were observed experimentally in the separate ranges of frequency. It makes more reliable our description of the data for EBPA where both relaxations overlap and had to be separated mathematically.

It is interesting that for MBBA as well as for EBPA the rotation round the long axis of the whole molecules is established to be the faster process.

\section{Conclusions}

Convergence of the performed interpretation for MBBA and EBPA results inclines to assume that the data collected for OH-MBBA and EBBA can be described quite analogously.

Arora et al. [7] observed during the heating of solid phase of OH-MBBA a "surprising" shift of the absorption maximum towards lower frequencies. It seems that their interpretation of this fact is not correct as no proper attention was paid to the reduction of the absorption maximum on increase in temperature from $-15^{\circ} \mathrm{C}$ to $-5^{\circ} \mathrm{C}$. Probably, the $\varepsilon^{\prime \prime}(f)$ curves, obtained for two lowest temperatures in Fig. 4 are connected with rotation of molecules round the long axis and on further heating this process gradually disappears as was found for MBBA and EBPA. 
The next two curves $\varepsilon^{\prime \prime}(f)$ received at the temperature $5^{\circ} \mathrm{C}$ and $15^{\circ} \mathrm{C}$ are probably connected with the rotation of the methoxy groups as this rotation should be slower than the rotation of the whole molecule as in MBBA and EBPA compounds. Thus, it seems that for OH-MBBA we can interpret the "surprising" reduction of maximum frequency as an occurrence of two processes at somewhat different timescale. On increasing temperature these processes are observed at higher frequencies, and the second faster process disappears gradually. Next, it was found that the $\varepsilon^{*}(f, T)$ results observed by Agarval et al. [8] in metastable solid phase of EBBA can be explained in the similar way. Their hypothesis of transformation of the nematic into glass was not confirmed in the newer measurements. Two maxima shown in Fig. 5 can be explained in accordance with interpretation proposed above: smaller maxima occurring in the lower temperature come from the rotation of molecules round a long axis, and higher maxima at higher temperature come from the rotation of ethoxy groups. Like for all other compounds also in the metastable phase of EBBA the rotation of the whole molecules is faster than the rotation of the ethoxy group. Thus, the above interpretation gives the uniform picture of the dynamics of molecules in four Schiff bases compounds discussed in this paper.

\section{Acknowledgments}

The work was partially supported by grant No. 2 P03B 02618 of the State Committee for Scientific Research.

\section{References}

[1] G. Pepy, R. Fouret, M. More, L. Rosta, Phys. Scr. 39, 485 (1989).

[2] R. Fouret, A. Elouatib, C. Gors, M. More, G. Pepy, L. Rosta, Phase Transit. 33, 209 (1991).

[3] J.K. Mościcki, Solid State Commun. 20, 481 (1976).

[4] J.K. Mościcki, S. Urban, Mol. Cryst. Liq. Cryst. 59, 73 (1980).

[5] J. Mayer, T. Waluga, J.A. Janik, Phys. Lett. A 41, 102 (1972).

[6] K. Pathmanathan, L.A. Dissado, R.M. Hill, Mol. Cryst. Liq. Cryst. 135, 65 (1986).

[7] V.P. Arora, V.K. Agarwal, A. Mansingh, J. Chem. Phys. 68, 4860 (1978).

[8] V.K. Agarwal, V.P. Arora, Mol. Cryst. Liq. Cryst. 45, 117 (1978).

[9] A. Bąk, A. Kocot, M. Massalska-Arodź, Proc. SPIE 4017, 207 (1999). 